\title{
Radiation enhancement of laser palliation for malignant dysphagia: a pilot study
}

National Medical Laser Centre and Department of Radiotherapy, University College Hospital, London I R Sargeant L A Loizou J S Tobias G Blackman $S$ Thorpe S G Bown

Correspondence to: Dr I R Sargeant, National Medical Laser Centre, Rm 103, The Rayne Institute, University Street, London WCIE 6JJ.

Accepted for publication 31 May 1992

\author{
I R Sargeant, L A Loizou, J S Tobias, G Blackman, S Thorpe, S G Bown
}

\begin{abstract}
Laser therapy offers rapid relief of dysphagia for patients with cancers of the oesophagus and gastric cardia but repeat treatments are required approximately every five weeks to maintain good swallowing. To try to prolong the treatment interval, 22 elderly patients were given additional external beam radiotherapy. Nine had squamous cell carcinoma and 13 adenocarcinoma: five had documented metastases. Six received $40 \mathrm{~Gy}$ and $16,30 \mathrm{~Gy}$ in 10 20 fractions. A 'check' endoscopy was performed three weeks after external beam radiotherapy. Dysphagia was graded from $0-4$ ( 0 =normal; $4=$ dysphagia for liquids). The median dysphagia grade improved from 3 to 1 after laser treatment. This improvement was maintained in the $30 \mathrm{~Gy}$ group but there was a noticeable deterioration in three of those who had received the higher radiation dose. A lifelong dysphagia grade of 2 or better was enjoyed by 14 of 16 patients in the $30 \mathrm{~Gy}$ group but only two of six in the $40 \mathrm{~Gy}$ group. The dysphagia controlled interval was 9 weeks (median) after check endoscopy and subsequent endoscopic procedures were required every 13 weeks to maintain good swallowing. There were no endoscopy related complications. Combined treatment is a promising approach for reducing the frequency of endoscopic treatments. The $30 \mathrm{~Gy}$ dose seems more appropriate and may prolong survival. A randomised study to test these conclusions is in progress.
\end{abstract}

(Gut 1992; 33: 1597-1601)

Carcinomas of the oesophagus and gastric cardia become symptomatic late in their natural history and commonly occur in the elderly who are unfit for surgery. Consequently, around $60 \%$ of these patients are deemed unsuitable for a curative attempt with surgery or radical radiotherapy at presentation. ' There are now many treatments available for palliation of this difficult condition. Laser treatment offers rapid and effective relief of dysphagia, it can be performed as an outpatient procedure, it does not have systemic effects, and serious complications occur in only about $1 \%$ of procedures. However, one major drawback of laser is the need for repeat treatments every five weeks or so in most patients to maintain good swallowing. ${ }^{2-4}$ Although laser is effective at tumour debulking, disease remains in the oesophageal wall and beyond the lumen in local nodes and thus tumour regrowth occurs fairly rapidly. Radiotherapy, however, has the potential for treating all the oesophageal tumour and the local regional draining sites ${ }^{5}$ and, thus, should be complimentary to laser. Further clinical data to support this argument have been outlined in a recent review."

External beam radiotherapy alone relieves dysphagia slowly, often taking several weeks for maximal effect. ${ }^{7}$ A recent study of palliative radiotherapy ${ }^{x}$ suggested that patients with relatively good swallowing enjoy improved survival over those who swallow poorly at presentation. There is, thus, both theoretical and clinical evidence to support the view that a patient whose swallowing has been improved by laser recanalisation should benefit further from radiotherapy. There has, however, been only one report ${ }^{y}$ to date in which the combination of laser and radiotherapy has been studied and intraluminal rather than external beam radiotherapy was used. Intraluminal radiotherapy (brachytherapy) causes superficial damage to the tumour as there is a rapid fall off in dose with distance from the source and it is not as effective in terms of irradiating the whole tumour as external beam treatment. The authors did, however, report benefit in terms of a prolonged 'dysphagia free interval' with additional brachytherapy in patients with squamous cell oesophageal cancers recanalised with laser. The present study was initiated to determine if palliative external beam radiotherapy, a more widely available and practical technique, reduces the need for frequent follow up procedures after laser treatment alone. All patients with a technically satisfactory result and who could swallow fluids or better after laser treatment were considered for additional radiotherapy.

\section{Methods}

\section{PATIENT SELECTION}

The laser unit at University College Hospital acts as a tertiary referral centre for patients suffering from malignant dysphagia who are considered unsuitable for surgery. A smaller number of patients treated with laser (around 20\%) present directly to the hospital. Patients recruited into this study were initially seen by us between September 1988 and June 1989. Patients with predominantly exophytic carcinomas of the oesophagus and gastric cardia thought suitable for laser treatment were eligible for the study. All patients recruited were deemed inoperable either because of advanced disease or because they presented an unacceptable anaesthetic risk. Five patients had documented metastatic disease, five had advanced local disease detected at computed tomography, and three had undergone laparotomy and their tumours could not be resected. A further nine were considered unsuitable for surgery because of age or general debility, or both. Patients with a good technical result from 
TABLE I Demographic patient data

\begin{tabular}{ll}
\hline Total no & 22 \\
Male/female & $15 / 7$ \\
Mean (SD) age (years) & $68(11)$ \\
Squamous call carcinoma/adenocarcinoma & $9 / 13$ \\
Cardia/thoracic & $15 / 6(1$ anastomotic) \\
Mean (SD) tumour length (cm) & $7(3)$ \\
Metastases & 5 \\
Inoperable - CT or at Laparotomy & 8 \\
Medically unfit & 9 \\
\hline
\end{tabular}

$C T=$ computed tomogram

laser treatment who swallow fluids, or better, were assessed for radiotherapy by a consultant radiotherapist (JST) before trial entry. Those who had had previous radiotherapy were excluded. All patients had either squamous cell carcinoma of the oesophagus or adenocarcinoma of the cardia. Patients with malignancy arising in organs other than the oesophagus and that caused dysphagia by direct invasion or metastatic spread were excluded. Full demographic data are given in Table I.

\section{Ethical aspects}

All patients were told about the nature of the study. Formal ethical committee approval was not considered necessary as both treatments are well established therapies for oesophageal cancer and all patients entered into the study were treated identically (no randomisation).

\section{ENDOSCOPIC TECHNIQUE}

The laser technique has been published in detail elsewhere. ${ }^{13}$ Treatment was performed under sedation with diazemuls/pethidine using an Olympus 1T 20 endoscope (KeyMed Ltd, Southend, UK). A flexilase Nd: YAG laser (Living Technology, Glasgow) was used in conjunction with a delivery system comprising a $0.4 \mathrm{~mm}$ quartz fibre contained in a $2.2 \mathrm{~mm}$ Teflon catheter. The distal end of the fibre, which protrudes beyond the tip of the endoscope during treatment, is protected by a metal tip that allows a coaxial stream of gas to be passed around the fibre to cool the tip and clear the target of blood and debris. Venting of gas was achieved by means of a tube attached via a three way tap to the working channel of the endoscope. The gas was vented through an underwater drain to remove debris and then out of the endoscopy room through a specially designed extractor fan. It is usual practice to begin laser treatment at the distal end of the tumour and proceed proximally as laser induced swelling may prevent passage of the scope. In cases where the tumour could not be negotiated with the endoscope, a guide wire was passed and the oesophagus was dilated to $18 \mathrm{~mm}$ with Celestin dilators. Polypoid areas were vaporised shaving them back to within

TABLE II Dysphagia controlled interval (DCI) (weeks) after 'check' endoscopy for all patients and broken down according to history

\begin{tabular}{lccr}
\hline & $\begin{array}{l}\text { Squamous cell } \\
\text { carcinomas }\end{array}$ & Adenocarcinomas & All patients \\
\hline All patients (median (range)) & $17(1-170)$ & $9(2-26)$ & $9(1-170)$ \\
30 Gy dose group only & $20(3-170)$ & $11(2-23)$ & $13(2-170)$ \\
Historical 'laser only' controls & 5 & 5 & 5 \\
\hline
\end{tabular}

2-3 mm of the oesophageal wall. Raised flat areas of tumour were coagulated and left to slough off (within a few days of treatment). Patients underwent laser sessions as appropriate to destroy intraluminal tumour and restore oesophageal patency before consideration for radiotherapy.

\section{RADIOTHERAPY}

All patients were irradiated using supervoltage teletherapy (cobalt 60 ). The target volume was determined by the length of tumour with a $5 \mathrm{~cm}$ margin at the upper and lower border of the tumour and a $3 \mathrm{~cm}$ margin circumferentially. The treatment was delivered by anterior and posterior opposed fields in all cases. In the early part of the study patients were initially given $20 \mathrm{~Gy}$ in five or 10 fractions and six patients who had tolerated this dose well were given a further $20 \mathrm{~Gy}$ dose either immediately or four to six weeks later (total dose $40 \mathrm{~Gy}$ ). It soon became apparent that most of these patients could not tolerate this total dose and subsequent patients all received $30 \mathrm{~Gy}$ in 10 fractions. Twenty patients had to stay in hospital for treatment. This increased the median lifetime total hospital stay to 21 days in comparison with historical inpatient times of around 14 days for laser only.

\section{FOLLOW UP}

All patients underwent follow up endoscopy three weeks after completing of radiotherapy (check endoscopy). Endoscopic findings were recorded as at the initial endoscopy, and further laser treatment was applied only to polypoid tumour. All patients were subsequently contacted monthly by the research sister (ST) to assess progress and the necessity for further treatment. The dysphagia score was recorded according to the scale previously used by us ( 0 =normal; $1=$ most solids; $2=$ semi-solids; 3 =liquids only; $4=$ difficulty with liquids). Patients whose dysphagia grade deteriorated by one or more points or who felt that their swallowing had deteriorated significantly were reendoscoped for assessment and further therapy as appropriate.

\section{Results}

\section{DYSPHAGIA GRADES}

The median dysphagia grade for all patients before laser treatment was 3 (liquids only) and this improved to a median of 1 (most solids) after laser treatment. The spectrum of dysphagia at presentation was very similar in patients treated with radiotherapy at the $30 \mathrm{~Gy}$ and $40 \mathrm{~Gy}$ dosage levels. After radiotherapy there was very little change in the dysphagia grades in the group treated with $30 \mathrm{~Gy}$ but three of the six receiving $40 \mathrm{~Gy}$ showed a noticeable deterioration and could swallow only fluids until their death. If a mean dysphagia grade of 2 (semi-solid diet) for the duration of each patient's survival is regarded as a success, 14 of 16 in the 30 Gy group and two of six in the $40 \mathrm{~Gy}$ group enjoyed a successful outcome. This difference is significant at the $5 \%$ level $\left(\chi^{2}\right.$ test $)$. 
TABLE III Survival data according to subgroup

\begin{tabular}{lccc}
\hline & Laser $+30 G y$ & Laser $+40 G_{y}$ & Laser only \\
\hline Patient no & 16 & 6 & +3 \\
Median (range) survival (weeks) & $+4\left(8-170^{\star}\right)$ & $19(7-73)$ & $22(+117)$ \\
l year survivors & $5 / 16\left(30^{\circ}\right)$ & $1 / 6\left(16^{\circ}\right)$ & $5 / 43\left(12^{\circ}\right)$ \\
\hline
\end{tabular}

*One patient still alive at 170 weeks (see text).

Three patients entered into this study were swallowing only fluids after initial laser recanalisation. One received the higher radiotherapy dose. Although radiotherapy was tolerated well by two of them, swallowing did not improve with treatment. One was eventually intubated and the other two enjoyed only short survival (eight and nine weeks respectively).

\section{DYSPHAGIA CONTROLLED INTERVAL AND NECES- SITY FOR REPEAT ENDOSCOPY}

The dysphagia controlled interval for the purpose of this study was the time between the 'check' endoscopy and the next follow up endoscopy, performed when the patient complained of further deterioration of swallowing, or to death if the patient never required another endoscopy. The figures for all patients broken down according to histology and radiotherapy dosage are shown in Table II and data from historical laser only patients ${ }^{2}$ are also given. Overall, patients treated with radiotherapy have a dysphagia controlled interval of 9 weeks (median) and for patients receiving the $30 \mathrm{~Gy}$ dose this is slightly longer (11 weeks). The dysphagia controlled interval was even longer (median 17 weeks) in patients with squamous cell carcinomas, although numbers are small.

Eleven patients required no further treatment after check endoscopy (one is still alive) and the median survival in this group was 9 weeks. Considering all those who required further endoscopic treatment after the check endoscopy, the median interval between follow up procedures required was 13 weeks (range 4-67). Eight patients required dilatation at check endoscopy but only three of these had fibrous strictures only, the rest having regrowth of tumour as well. Overall, 13 patients required further endoscopic treatment for tumour after radiotherapy. Three

Figure 1: Survival curves, laser $(Y A G)+30 G y$ radiotherapy and historical laser only controls.

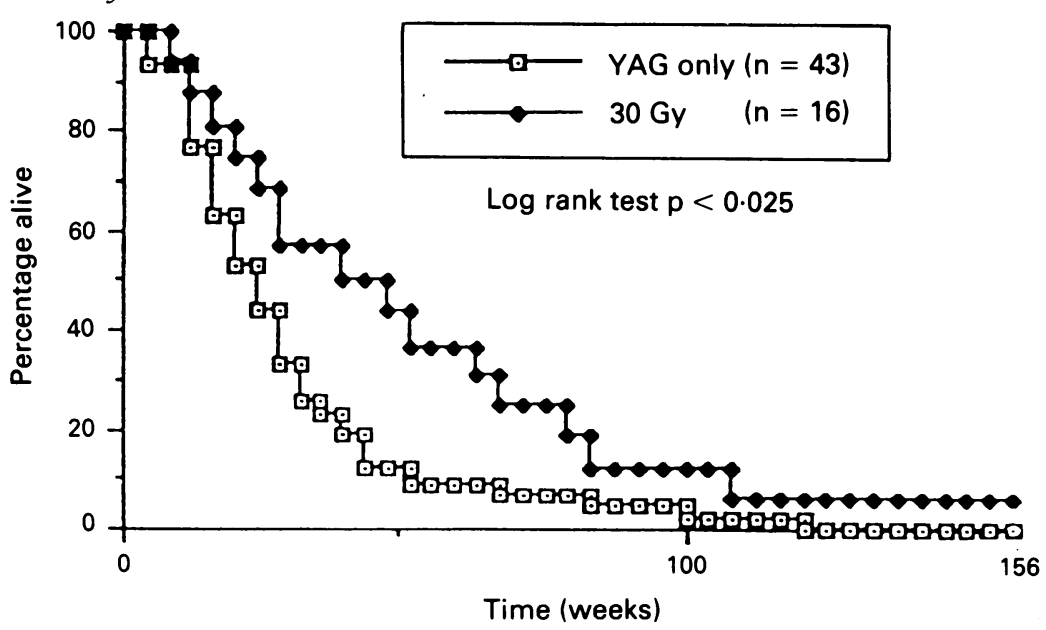

months, and 14 months after start of treatment). All swallowed well with tubes (dysphagia grade 2). Two survived several more months but the third died a few weeks after intubation. All but one of the patients have died at the time of writing. None died of aspiration, all gradually weakened with cancer cachexia or the effects of metastatic disease, or both.

SURVIVAL

This study was not set up to assess survival but it became apparent that patients in the $30 \mathrm{~Gy}$ group were doing well and it was decided to examine survival data in detail. Table III shows the crude survival data from presentation for each group receiving radiotherapy and for historical laser only controls, again taken from our earlier study.' These data give the impression of improved survival in the $30 \mathrm{~Gy}$ group and therefore survival curves were plotted (Figs 1 and 2 ). These curves were analysed using the log rank test and this shows a significantly $(p<0.025)$ prolonged survival in the group receiving the 30 Gy dose compared with historical controls. As the study was not randomised it could reasonably be argued that this difference is at least partly due to differences in selection. There was no statistical difference between the groups receiving different doses of radiotherapy, although the survival of patients receiving $40 \mathrm{~Gy}$ is generally shorter. One patient in the 30 Gy group is still alive. She is a 78 year old who presented with a 3 $\mathrm{cm}$ squamous cell cancer and has remained well for 170 weeks with entirely normal swallowing since treatment at three laser sessions followed by the radiotherapy.

\section{COMPLICATIONS}

No perforations were experienced in this group of patients. Mild nausea, lethargy, and odonyphagia were common during radiotherapy but usually did not amount to more than a minor irritation for most patients. Radiotherapy was poorly tolerated in three of the six patients who received the higher radiotherapy dose. They never really recovered after radiation, most did not swallow well and succumbed fairly rapidly from cachexia. Only two of the 16 who received the 30 Gy dose had more than minor symptoms. One of these succumbed early with poor swallowing but the other recovered and eventually died swallowing well 24 weeks after treatment.

Fibrous strictures were identified in three patients at the 'check' endoscopy and another five had fibrous narrowing as well as further luminal tumour growth. Two of those with fibrous strictures required no further treatment after dilatation at the 'check' procedure and the third needed dilations every 9 weeks for a year.

\section{Discussion}

There are many techniques available for the palliation of patients with cancer of the oesophagus and gastric cardia. In addition to the $\mathrm{Nd}$ YAG laser, these include intubation with prosthetic tubes, external beam radiotherapy, and 


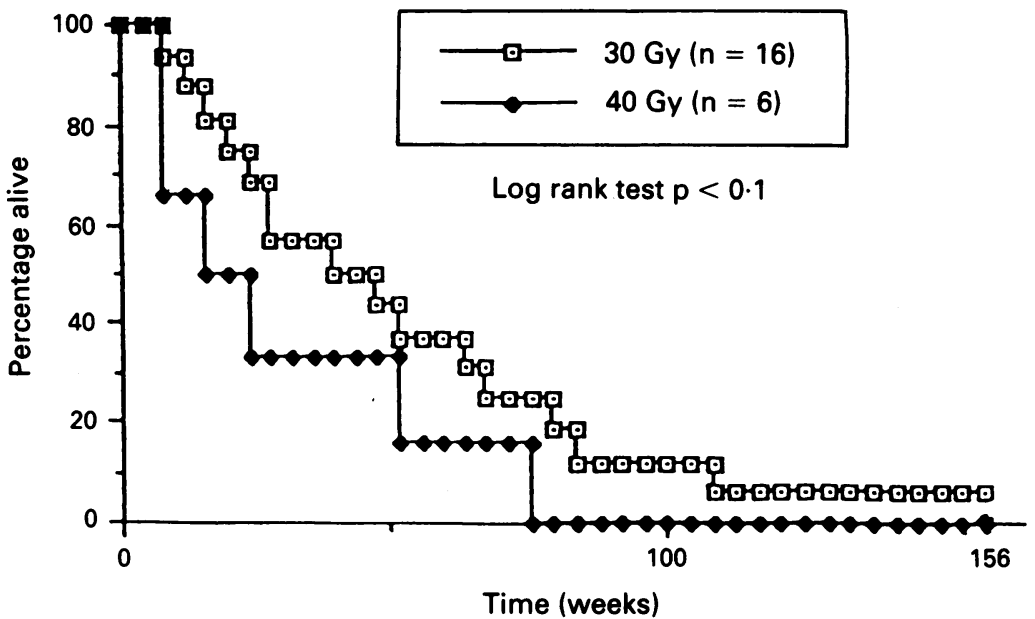

Figure 2: Survival curves, laser $(Y A G)+30 G y$ radiotherapy and laser +40 Gy radiotherapy.

intracavitary radiotherapy (brachytherapy). The important parameters to consider in assessing these techniques are the quality of swallowing achieved, which is well reflected in the overall quality of life $\mathrm{e}^{24}$ and the price paid to achieve that quality (number of endoscopies, time in hospital for procedures, procedure related complications). Intubation, while offering rapid relief, does not allow many patients to take solids. ${ }^{2}$ In addition, there is a higher risk of procedure related perforation than with laser $(13 \% v 2 \%$ for laser in a series at this unit $\left.{ }^{2}\right)$. Potentially serious long term complications of tube are reported in all series and comprise tube displacement (3$19 \%)$, overgrowth (2-7\%), and late perforation $(0-7 \%){ }^{2411-13}$ Brachytherapy ${ }^{14}$ looks promising but can cause severe oesophagitis and little data are yet available on the quality of palliation.

It is only recently that researchers have started to compare techniques in similar patient groups. A study from our unit comparing laser with intubation ${ }^{2}$ has given us a better idea of which patients are likely to benefit most in terms of quality of swallowing from each of these methods. Those patients who do well with laser achieve a quality of swallowing that is better than the best attainable by tube. The quality of swallowing achieved with a tube is much more consistent (semi-solid diet) but only a few patients can swallow any solids. The availability of two treatments can thus be used to maximise palliation in individual patients. In our unit it is now standard policy to intubate patients who fail to manage at least a semi-solid diet with laser therapy.

In order to improve further on the results using different techniques in sequence we have elected to combine treatments. This study was designed to give an indication of whether the theoretically attractive combination of laser and external beam radiotherapy is clinically useful and if so at what dose. More specifically, the hope was to combine the better palliation of dysphagia with laser with more prolonged relief normally achievable only with a tube. The results look promising, although $50 \%$ of patients require follow up procedures after the 'check' endoscopy the frequency of these seems to be reduced quite dramatically in comparison to historical control data.

As these patients are receiving palliative treat- ment, it is important not to be so aggressive that the side effects of radiotherapy detract significantly from the benefit achieved with laser, either in terms of deterioration in general condition or dysphagia. The results indicate that the $30 \mathrm{~Gy}$ dose in 10 fractions is well tolerated in this patient group. Our limited experience of higher doses in this palliative setting is not favourable, although the numbers treated were small. Overall, the dysphagia controlled interval is prolonged and the subsequent necessity for follow up endoscopy is reduced, particularly for squamous cell cancers in comparison to historical control data. It is of interest that the brachytherapy study mentioned ${ }^{9}$ showed a prolonged 'dysphagia free' interval but only for patients with squamous cell tumours. One concern of applying two treatments which can induce fibrosis was that we would have an unacceptable number of fibrous strictures but that was not the case. Only three patients came back with sole 'fibrous' narrowing at the check endoscopy. Overall, 13 patients required further laser for tumour after external beam radiotherapy. This suggests that the radiotherapy may be slowing tumour regrowth rather than causing wholesale tumour necrosis.

The survival curves for the $30 \mathrm{~Gy}$ group and 43 historical controls indicate the possibility of increased survival with external beam radiotherapy. However, it is important to treat comparisons with historical data with caution in view of possible differences in patient selection.

The results of this pilot study are promising and indicate that a randomised study to evaluate the combination of laser and external beam radiotherapy at the $30 \mathrm{~Gy}$ dose in 10 fractions in a larger number of patients would be worthwhile. Such a study is already underway. In view of others' experience ${ }^{8}$ and our own with patients with dysphagia grade 3 who received radiotherapy, we have elected to randomise only patients who are able to swallow a semi-solid diet, or better, after laser.

Other endoscopic techniques for relieving swallowing such as alcohol injection or BICAP probe have been shown effective in the palliation of malignant dysphagia. ${ }^{14}$ is It is likely that any benefit seen with radiotherapy in combination with laser would be mirrored for such techniques and appropriate studies with these combinations should be encouraged.

All authors were supported by the Department of Health Special Medical Development on Lasers.

1 Watson A. Surgery for carcinoma of the oesophagus. Postgraduate Medical Fournal 1988; 64: 860-4.

2 Loizou LA, Grigg D, Atkinson CB, Robertson C, Bown SB. A Prospective Comparison of Laser Therapy and Intubation in Endoscopic Palliation for Malignant Dysphagia. Gastroenterology 1991; 100: 1303-10.

3 Krasner N, Barr H, Skidmore C, Morris AI. Palliative laser therapy for malignant dysphagia. Gut 1987; 28: 792-8.

4 Barr H, Krasner N, Roouf A, Walker RJ. Prospective randomised trial of laser therapy only and laser therapy followed by endoscopic intubation for the palliation of malignant dysphagia. Gut 1990; 31: 252-8.

5 Tobias JS, Bown SG. Palliation of malignant obstruction - use of lasers and radiotherapy in combination. European fournal of Cancer 1991; 27: 1350-52.

6 Bown SG. Palliation of malignant dysphagia: surgery, radiotherapy, laser, intubation alone or in combination? Gut 1991; 32: $841-4$.

7 Pearson JG. Present status and future potential of radiotherapy in the management of oesophageal cancer. In: Silber F, ed. Carcinoma of the oesophagus. Rotterdam: Balkema, 1978: 334-9. 8 Caspers RJL, Welvaart K, Verkes J, Hermans J, Leer JWH. 
The effect of radiotherapy on dysphagia and survival in patients with oesophageal cancer. Radiotherapy and oncology $1988 ; 12: 15-23$.

9 Sander R, Hagenmueller F, Sander C, Reiss G and Classen M. Laser versus laser plus afterloading with iridium 192 in the palliative treatment of malignant stenosis of the oesophagus: a prospective, randomised, and controlled study. Gastrointestinal Endoscopy 1991; 37: 433-40.

10 Ogilvie AL, Dronfield MW, Ferguson R, Atkinson M Palliative intubation of oesophagogastric neoplasms at fibreoptic endoscopy. Gut 1982; 23: 1060-67.

11 Tytgat GNJ, Bartelsman JFWM, Den Hartog Jager K Tytgat GNJ, Bartelsman JFWM, Den Hartog Jager K,
Mathus-Vliegen EMH. Upper Intestinal and Biliary Tract Endoprosthesis. Dig Dis Sci 1986; 31: 57S-76S.

12 Gasparri G, Casalegno PA, Camandona M. Endoscopic in sertion of 248 prosthesis in inoperable carcinoma of the oesophagus and cardia: short-term and long-term results Gastrointestinal Endoscopy 1987; 33: 354-6.

13 Loizou LA, Bown SG. Clinical laser applications in the G tract. In: Pounder R. Recent advances in Gastroenterology. Edinburgh: Churchill Livingstone: 1988.

14 Rowland CG, Pagliero KM Intracavitary irradiation in pallia tion of carcinoma of the oesophagus and cardia. Lancet 1985; ii: 981-3.

15 Payne-James JJ, Spiller RC, Misiewicz JJ, Silk DBA. Use of ethanol-induced tumor necrosis to palliate dysphagia in patients with esophagogastric cancer. Gastrointestinal Endoscopy 1990; 36: 43-6.

16 Jensen DM, Gustavo M, Randall G, Tung LA, English-Zych S. Comparison of low-power YAG laser and BICAP tumor probe for palliation of oesophageal cancer strictures. Gastroenterology 1988; 94: 1263-70.

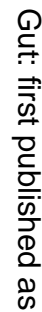

